\title{
Assessment of hyperinflation in children with cystic fibrosis
}

\author{
J Marchant, D M Hansell, A Bush
}

\begin{abstract}
Background - Radiological estimates of hyperinflation are used in several clinical and radiographic scoring systems for cystic fibrosis, but it is not known if these estimates of hyperinflation are related to measured total lung capacity.

Methods - Comparison was made of independent clinical estimates of hyperinflation from chest radiographs with objective plethysmographic and radiographic measurements of total lung capacity in $\mathbf{2 5}$ children with cystic fibrosis.

Results - There was good agreement between plethysmographic and radiographic measurements. Clinical estimation correctly predicted the extremes of hyperinflation, but grading was no more than $50 \%$ accurate in all other groups.

Conclusion - The degree of hyperinflation cannot be estimated by inspecting chest radiographs in many children with cystic fibrosis. This does not invalidate the scoring systems, but suggests that a better term than "hyperinflation" should be sought.
\end{abstract}

(Thorax 1994;49:1164-1166)

The commonly used quantitative scoring systems for the chest radiograph in cystic fibrosis require scoring of the degree of hyperinflation. ${ }^{12}$ For example, the Brasfield score ${ }^{2}$ grades air trapping from 0 (absent) to 4 (most severe) on the basis of overdistension manifest by sternal

13 June

Accepted for

16 June 1994

Individual patient dato

\begin{tabular}{|c|c|c|c|c|c|}
\hline \multirow[t]{2}{*}{$\begin{array}{l}\text { Patient } \\
\text { no. }\end{array}$} & \multirow[t]{2}{*}{$\begin{array}{l}T L C \\
\text { pred }(m l)\end{array}$} & \multirow{2}{*}{$\begin{array}{l}T L C \text { chest } \\
\text { radiography } \\
\text { Actual (\% pred) }\end{array}$} & \multirow{2}{*}{$\begin{array}{l}\text { TLC plethysmo- } \\
\text { graphy } \\
\text { Actual (\% pred) }\end{array}$} & \multirow[t]{2}{*}{ Observer 1} & \multirow[t]{2}{*}{ Observer 2} \\
\hline & & & & & \\
\hline 1 & 4526 & $4690(104)$ & $4390(97)$ & 0 & 1 \\
\hline 2 & 6065 & 7951 (131) & $6450(108)$ & 2 & 2 \\
\hline 3 & 5052 & 5980 (119) & $4850(96)$ & 1 & 2 \\
\hline 4 & 6196 & 7249 (117) & 6630 (107) & 1 & 0 \\
\hline 5 & 1792 & 2628 (147) & $2150(120)$ & 2 & 2 \\
\hline 6 & 2860 & 3560 (124) & 3260 (114) & 3 & 3 \\
\hline 7 & 4329 & $4746(110)$ & $3420(79)$ & 3 & 3 \\
\hline 8 & 4614 & 4663 (101) & $3830(83)$ & 1 & 3 \\
\hline 9 & 2186 & $2684(122)$ & $2230(102)$ & 1 & 2 \\
\hline 10 & 2402 & $1727(72)$ & $2330(97)$ & 1 & 2 \\
\hline 11 & 1816 & $2813(155)$ & 2670 (147) & 4 & 4 \\
\hline 12 & 3176 & $3113(98)$ & 3350 (105) & 1 & 3 \\
\hline 13 & 2485 & $3587(144)$ & $3280(132)$ & 2 & 3 \\
\hline 14 & 2896 & 3954 (137) & $3620(125)$ & 3 & 3 \\
\hline 15 & 3804 & $5423(123)$ & $3500(92)$ & 2 & 3 \\
\hline 16 & 1958 & 2508 (128) & $2350(120)$ & 0 & 2 \\
\hline 17 & 3765 & 3994 (106) & $3690(98)$ & 2 & 3 \\
\hline 18 & 3151 & 3547 (113) & $2710(86)$ & 2 & 3 \\
\hline 19 & 3418 & 4486 (132) & 3350 (98) & 2 & 3 \\
\hline 20 & 2558 & 4358 (171) & 3300 (129) & 4 & 4 \\
\hline 21 & 5056 & 5935 (118) & $4500(89)$ & 1 & 1 \\
\hline 22 & 4058 & 4470 (110) & $4180(103)$ & 3 & 3 \\
\hline 23 & 1805 & $2350(130)$ & 2040 (113) & 3 & 2 \\
\hline 24 & 3186 & 3231 (101) & $3250(102)$ & 1 & 2 \\
\hline 25 & 1906 & 2200 (115) & 2440 (128) & 0 & 2 \\
\hline
\end{tabular}

$\mathrm{TLC}=$ total lung capacity; observer $=$ clinical score $(0-4)$. bowing, depression of diaphragms, and/or thoracic kyphosis. However, no study has compared estimates of hyperinflation with simultaneous objective lung function data to see if such estimates are valid.

\section{Methods}

Twenty five children (16 boys) of mean age 11.4 (range 5.9-16.6) years from the paediatric cystic fibrosis clinic were studied. The diagnosis had been established on the basis of a compatible history and duplicate sweat sodium levels of more than $70 \mathrm{mmol} / \mathrm{l}$ on at least $100 \mathrm{mg}$ sweat. As part of their routine annual assessment they underwent, in random order on the same day, posteroanterior and lateral chest radiography at full inspiration taken under known conditions of magnification, and whole body plethysmography.

Total lung capacity was estimated by digitising the chest radiographs $s^{3-5}$ by the same observer (JM); this involves using a digitising tablet linked to a personal computer to measure the dimensions of the chest wall, heart, mediastinal, and subdiaphragmatic structures from posteroanterior and lateral chest radiographs. Allowance is made for magnification. The algorithms use shape assumptions to measure lung volume to a good degree of accuracy. Care is necessary to ensure that the patient has taken a full inspiration. Total lung capacity was also recorded using a Fenyves and Gut whole body plethysmograph in the routine lung function laboratory (technical staff) taking the mean of three measurements required to be within $5 \%$ of each other. Hyperinflation was scored as none, minimal, moderate, marked, severe and graded $0-4^{2}$ independently by a radiologist (DH) and a paediatric respiratory physician (AB) using the standard criteria. ${ }^{12}$ All measurements and assessments were made without knowledge of the other results.

Results

The results of plethysmographic and radiographic measurement of total lung capacity were very similar (radiographic lung volume $(\mathrm{ml})=1.23 \times$ plethysmographic lung volume $-224, r^{2}=0.88$; table). Radiographic estimation of lung volume was a mean of $563 \mathrm{ml}$ greater than plethysmographic lung volume. Hyperinflation was defined as total lung capacity above the normal range for our laboratory - that is, $>115 \%$ predicted. Mild, medium, marked, and severe hyperinflation were arbitrarily defined as $115-125 \%, 125-135 \%$, $135-145 \%$, and $>145 \%$ of predicted total lung capacity. Although the two observers 


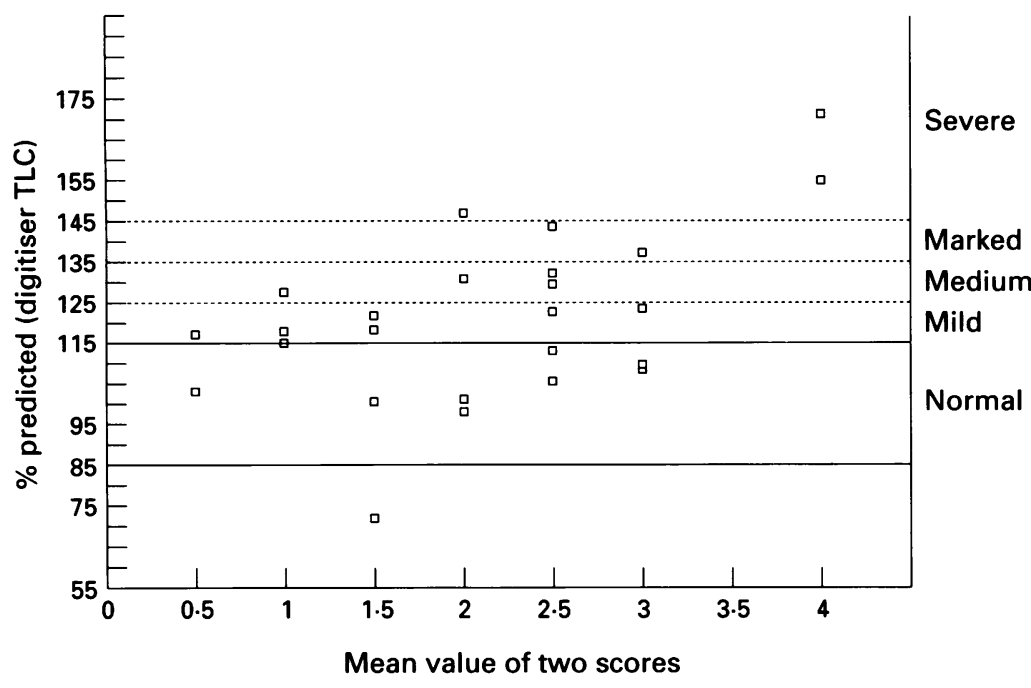

Figure 1 Comparison between the mean of the two clinical scores and total lung capacity (TLC) measured from the digitiser. Although the extremes are correctly graded, there is poor agreement between clinical score and plethysmographic measurement.

agreed to within a single grade in 22 of 25 children (table), overall concordance was poor $($ kappa $=0 \cdot 19)$. Both observers assigned the patients with mild and severe hyperinflation to the correct grades defined by plethysmographic and radiographic measurements. No patient with severe hyperinflation was incorrectly graded. In all other groups, however, neither observer scored with more than $50 \%$ diagnostic accuracy. The mean score of both observers was no better than either alone (fig 1). The results were the same whether the observers were compared with plethysmographic or radiographic measurements of total lung capacity.

A

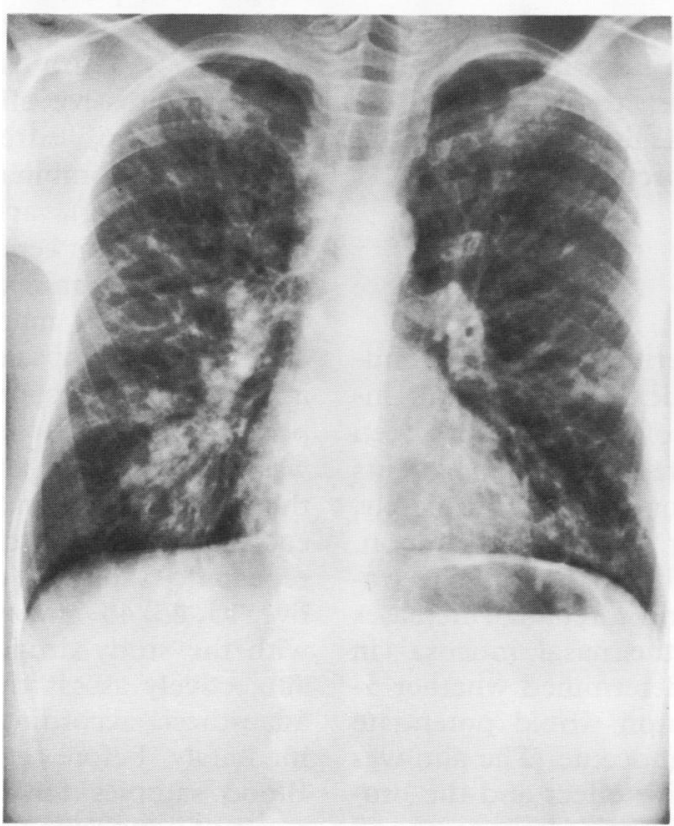

Discussion

Radiographic and plethysmographic estimates of total lung capacity showed reasonable agreement in these patients with cystic fibrosis, similar to that found in other studies. ${ }^{3-5}$ The overestimate by radiography has been attributed to lung tissue volume, which is not measured by plethysmography. The main conclusion of this study, however, is that subjective assessment of hyperinflation from chest radiographs is unreliable, despite the good agreement between plethysmographic and radiographic values. Occasional radiographic estimates of total lung capacity are very different from plethysmography. We therefore checked our conclusions by repeating the comparisons with plethysmographic instead of radiological estimations. Comparison of radiographic estimates with plethysmography did not alter the conclusions. The allocation of degrees of hyperinflation to particular bands of lung volume was to some extent arbitrary; $115 \%$ predicted has been taken as the upper limit of normal, ${ }^{6}$ but different ways of dividing mild, moderate, and severe above this level did not significantly improve the results. Scoring systems for cystic fibrosis include an assessment of hyperinflation; from this study it is clear that, whatever is being assessed on the radiographs, it bears little relation to objective measurements of lung volume (fig 2 ). This does not necessarily invalidate the scoring systems, but does suggest that a better term should be sought and that clinicians should not delude themselves that they can objectively assess lung volumes from chest radiographs, at least in children with cystic fibrosis.

1 Shwachman H, Kulcyczki LL. Long term study of 105 patients with cystic fibrosis. Am $\mathcal{F}$ Dis Child 1958;96:6-15.

B

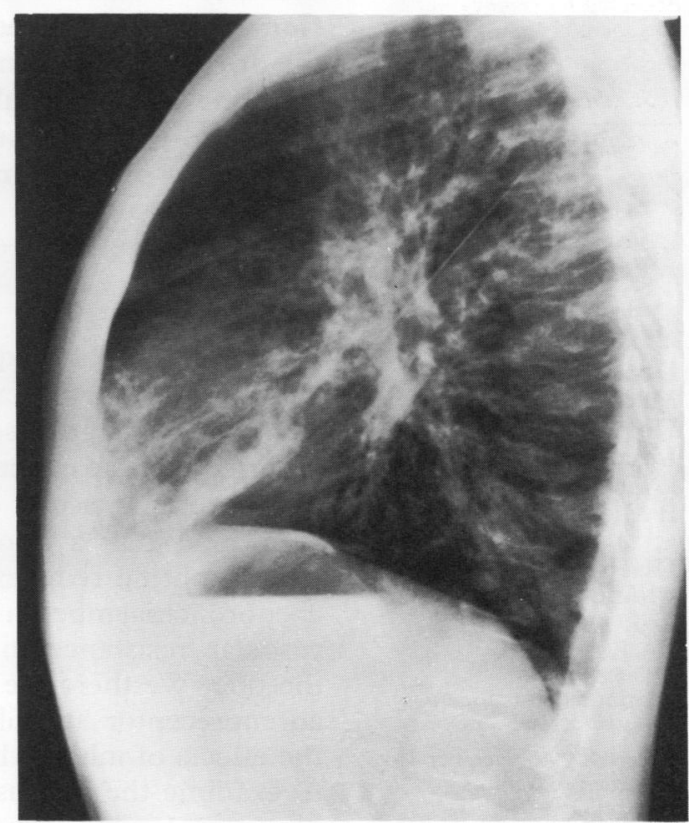

Figure 2 (A) Posteroanterior and (B) lateral chest radiographs scored as severely hyperinflated by both observers. Total lung capacity measured radiographically and plethysmographically was normal. 
2 Brasfield D, Hicks G, Soong SJ, Tiller RE. The chest roentgenogram in cystic fibrosis: a new scoring system. Pediatric 1979;63:24-9.

3 Pierce RJ, Brown DJ, Holmes M, Cumming G, Denison $D M$. The estimation of lung volume from chest radiographs using shape information. Thorax 1979;34:726-34.

4 Pierce RJ, Brown DJ, Denison DM. Radiographic, scin- tigraphic and gas-dilution estimates of individual lung and lobar volume. Thorax 1980;35:773-80.

5 Bush A, Denison DM. Use of different magnification factors to calculate radiological lung volumes. Thorax $1986 ; 41$ : 158-9.

6 Denison DM, du Bois R, Sawicka E. Pictures in the mind. Br $\mathcal{F}$ Dis Chest 1983;77:35-50.

\title{
Effects of inhaled lignocaine and adrenaline on capsaicin-induced cough in humans
}

\author{
L Hansson, B Midgren, J-A Karlsson
}

\begin{abstract}
Background - The hypothesis that adrenaline can augment and/or prolong the antitussive effect of nebulised lignocaine was examined.

Methods - The effect of inhaled lignocaine alone $(20 \mathrm{mg})$ and in combination with adrenaline $(400 \mu \mathrm{g})$ was studied on capsaicininduced cough in 10 healthy subjects.

Results - Cough was significantly reduced between five and 25 minutes by lignocaine. Adrenaline alone had no inhibitory effect and it neither augmented nor prolonged the antitussive effect of lignocaine. The subjective anaesthesia by lignocaine was short lasting (less than 15 minutes) and not altered by adrenaline, suggesting different sensory mechanisms for anaesthesia and cough suppression. Plasma concentrations of lignocaine were low $(<30 \mathrm{ng} / \mathrm{ml})$, not altered by adrenaline, and did not correlate with the local anaesthetic or the antitussive effect.

Conclusions - Lignocaine acts locally in the oropharynx and airways and adrenaline does not alter the effect or absorption of nebulised lignocaine on the human respiratory mucosa.
\end{abstract}

(Thorax 1994;49:1166-1168)

Department of Lung Medicine, University Hospital, S-221 85 Lund, Sweden

L Hansson

B Midgren

Discovery Biology, Rhône-Poulenc Rorer Ltd, London, UK J A Karlsson

Reprint requests to: Dr L Hansson.

Received 29 November 1993 Returned to authors 20 April 1994 Revised version received 27 June 1994 27 June 1994 19 July 1994 the effects of inhaled lignocaine. The aim was to examine the antitussive effect and the oropharyngeal numbness of inhaled lignocaine and adrenaline on capsaicin-induced cough in healthy human subjects. In addition, plasma levels of lignocaine were measured to determine the degree of systemic absorption.

\section{Methods}

Ten non-smoking healthy subjects (five women) of mean age 27 (range 18-33 years) took part in the study. They gave their written informed consent and the study was approved by the University Hospital medical ethics committee, Lund. Capsaicin (Sigma) was dissolved in ethanol and diluted with $0.9 \% \mathrm{NaCl}$ to 0.4 $\mu \mathrm{mol} / 1,2 \mu \mathrm{mol} / 1,10 \mu \mathrm{mol} / 1$, and $50 \mu \mathrm{mol} / 1$.

Capsaicin was inhaled by tidal breathing from a nebuliser (BIRD Asmastick, output $0.5 \mathrm{ml} / \mathrm{min}$ and mass median diameter $3 \mu \mathrm{m}$ ) filled with $2 \mathrm{ml}$ of solution. ${ }^{1}$ A microphone and tape recorder were used to register the sounds of breathing and cough. The number of coughs was counted from the tape recordings. Blood samples were drawn from an arm vein into heparinised tubes. All blood samples were centrifuged and plasma was then separated and stored at $-25^{\circ} \mathrm{C}$ until analysed by gas chromatography (Astra Alab, Södertälje, Sweden).

The antitussive effects of nebulised lignocaine $(20 \mathrm{mg})$, adrenaline $(400 \mu \mathrm{g})$, lignocaine in combination with adrenaline $(20 \mathrm{mg}+400 \mu \mathrm{g})$, and vehicle (saline) were studied on four separate study days. Treatments were administered in a randomised, double blind manner. Increasing concentrations of capsaicin $(0 \cdot 4-50 \mu \mathrm{mol} / \mathrm{l})$ were inhaled until a response of at least 10 coughs per minute was reached, and the concentration was then repeated and the mean response of the two challenges used as a baseline value. On each study day the chosen concentration was repeated. Capsaicin challenges were repeated five, $15,25,45$, and 60 minutes after treatment with the study drug. Subjects were asked to subjectively assess the level of oropharyngeal anaesthesia according to a five point scale immediately before each capsaicin challenge. Blood samples for determination of plasma levels of lignocaine were drawn before and 10, $20,30,45$, and 60 minutes after lignocaine inhalation. Kruskal-Wallis test was used at each 